\title{
Effect of magnetic field on hysteretic characteristics of silicon diodes under conditions of low-temperature impurity breakdown
}

\author{
A.B. Aleinikov ${ }^{1,3}$, V.A. Berezovets ${ }^{2,3}$, V.L. Borblik ${ }^{1}$, M.M. Shwarts ${ }^{1}$, Yu.M. Shwarts ${ }^{1,3}$ \\ ${ }^{1} V$. Lashkaryov Institute of Semiconductor Physics, NAS of Ukraine, \\ 41, prospect Nauky, 03028 Kyiv, Ukraine; \\ Phone/fax: +38 (044) 525-7463; *e-mail: borblik@isp.kiev.ua \\ ${ }^{2}$ Ioffe Physical-Technical Institute, Russian Academy of Sciences, 194021 Saint-Petersburg, Russia \\ ${ }^{3}$ International Laboratory of High Magnetic Fields and Low Temperatures, \\ 53-421, Wroclaw, Poland
}

\begin{abstract}
Effect of magnetic field (up to $14 \mathrm{~T}$ ) on current-voltage characteristics of silicon $n^{+}-p$ diodes which manifests hysteresis loops related with low-temperature impurity breakdown has been studied. With growth of magnetic field, the hysteresis loops are narrowed and decreased in amplitude and then disappear, but the breakdown continues in a soft form. Planar design of the diode has allowed separating the influence of magnetic field on mobility of the carriers executing impact ionization of the impurities and on the ionization energy itself. Theoretical analysis of the experimental data permitted us to determine the dependence of the ionization energy on the magnetic field. As in other investigated semiconductors, our results demonstrate the dependence of $B^{1 / 3}$ type. A model capable to explain qualitatively the mechanism of suppression of the hysteresis loops by magnetic field is proposed as well.
\end{abstract}

Keywords: $p-n$ diode, silicon, low temperature, impurity breakdown, hysteresis, magnetic field.

Manuscript received 23.07.12; revised version received 27.08.12; accepted for publication 10.09.12; published online 25.09.12.

\section{Introduction}

In silicon, as in a number of other semiconductors doped with shallow impurities, the concentration of which is below than the critical one for insulator-metal transition (IMT), freezing-out of free current carriers takes place at low temperatures. In relation with this fact, lowtemperature silicon conduction has hopping character and in electric fields of a sufficient amplitude, impact ionization of these impurities can occur, which is accompanied, in a number of cases, by hysteresis loops in the current-voltage characteristics (CVC). These hysteresis loops also manifests themselves in devices based on silicon: in diodes $[1,2]$, in field-effect transistors [3].

In Ref. [1], effect of magnetic field on impurity breakdown in commercial silicon diodes 1N4001-4005 was studied, where switching characteristics were observed within the temperature range of 15 down to $7 \mathrm{~K}$. The magnetic field (as low as $0.14 \mathrm{~T}$ ) perpendicular to the current suppressed the hysteresis loops. Conclusive interpretation of the effect was absent; the higher magnetic fields were not investigated.

Numerous investigations of magnetic field influence on low-temperature impurity breakdown in $p$ Ge and $n$-GaAs (see, for example, papers [4-6]) were concentrated on problems of beginning spontaneous current oscillations under these conditions and their transition to chaos. It is talked, as a rule, about low magnetic fields and slightly doped materials in the form of resistors. Only in papers [7, 8], the effect of magnetic field on hysteresis itself was described for $n$-GaAs.

In this paper, we report about measurements of low-temperature characteristics of experimental silicon $n^{+}-p$ diode that exhibits switching effect at temperatures below $27 \mathrm{~K}$ (measurements were performed down to $1.7 \mathrm{~K}$ ) in magnetic fields up to $14 \mathrm{~T}$. Design of the investigated diode was planar, with circular geometry of layers; the substrate was (111)-oriented Si. The doping level of the diode base (by boron) was close to the 

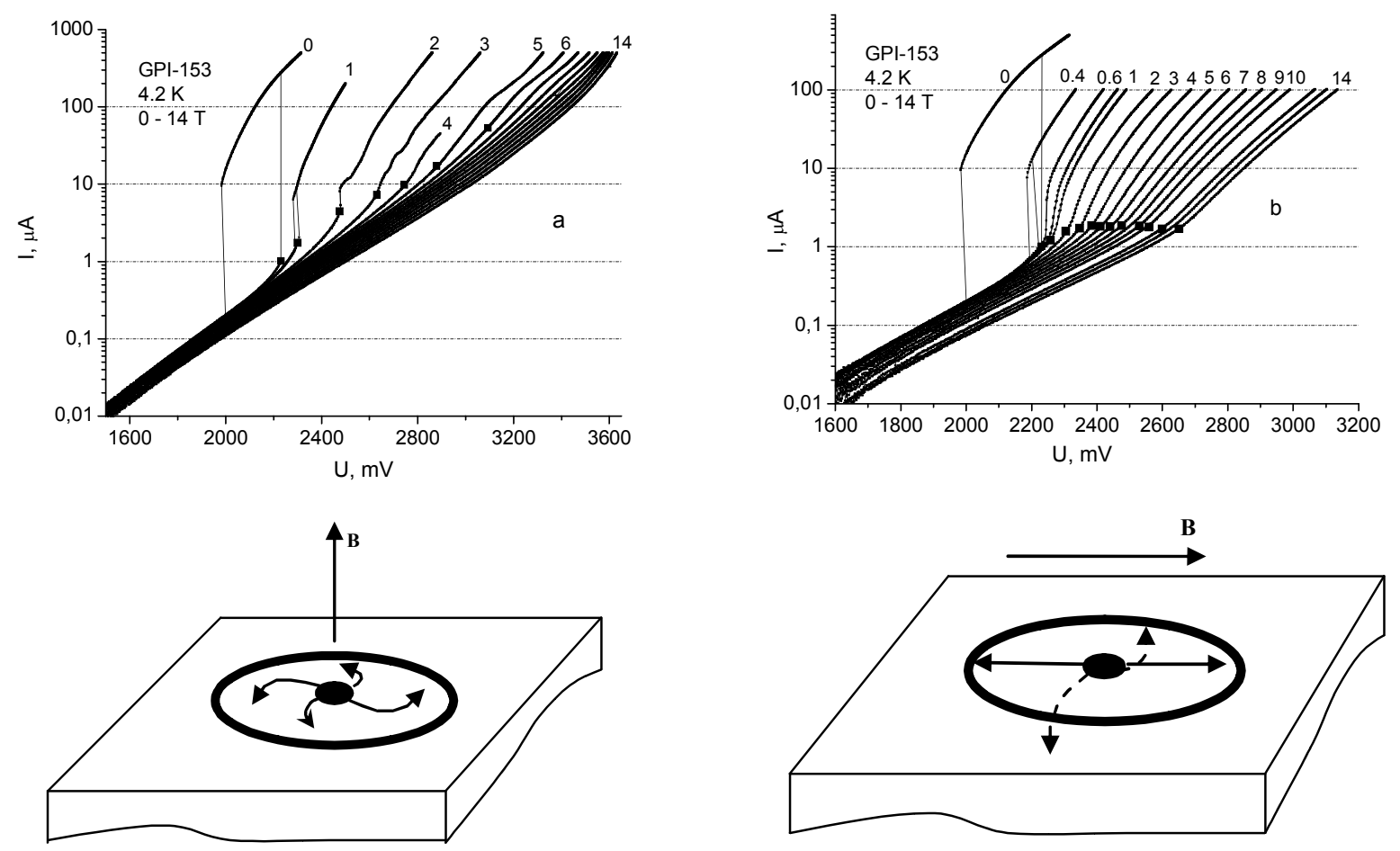

Fig. 1. Current-voltage characteristics of investigated diode at $4.2 \mathrm{~K}$ against magnetic induction as a parameter (numbers near the curves are its magnitude in Tesla): (a) - magnetic field is perpendicular to the diode structure plane, (b) - magnetic field is parallel to it; bold points on the curves indicate the thresholds of impact ionization.

critical value for IMT but remained on its insulator side; therefore at low temperatures, freezing-out of free carriers into impurities took place. Theoretical analysis of the experimental results has allowed determination of the magnetic-field dependence of ionization energy of boron impurity in silicon at high doping level. A qualitative model for explanation of magnetic suppression of hysteresis loops in the diode CVC is given as well.

\section{Experimental data}

The results of measurements of the diode CVCs at $4.2 \mathrm{~K}$ are represented in Fig. 1: for magnetic field oriented perpendicular to the diode structure plane (a) and for its orientation in parallel to the diode structure plane (b). The lower parts of Fig. 1 illustrate a character of motion for free electrons which are available in small number even at low temperatures and execute the impact ionization of the impurities. Note that under orientation of magnetic field parallel to the structure plane, a portion of the current lines is available, which coincides in direction with the magnetic field and, therefore, do not suffer action of the Lorentz force.

Under magnetic field orientation perpendicular to the structure plane, hysteresis loops in CVC narrow with growth of magnetic field decreasing in the amplitude and then disappear. The breakdown, however, remains in a soft form (corresponding thresholds are marked by bold points on the curves), and later it disappears as well. Under magnetic field orientation parallel to the structure plane, character of its action is substantially different. Disappearance of the hysteresis loops takes place earlier, in lower magnetic fields, but the soft breakdown, in contrast to previous case, remains up to the highest reached fields (14 $\mathrm{T})$.

As it is known, there are two main mechanisms of magnetic field action on the effect of impact ionization: 1) curling trajectories of carriers executing impact ionization that manifests itself in decreasing their mobility and 2) compressing wave functions of carriers localized on impurity center that manifests itself in the increasing ionization energy of the center (effect of magnetic freezing-out) ${ }^{1}$. It is obvious that in the case of perpendicular orientation, the first mechanism (decreasing electron mobility) dominates: in high magnetic fields, this decreasing is so significant that the breakdown disappears at all. In the case of parallel orientation, electron mobility does not vary with magnetic field; therefore the breakdown (in soft form) remains up to the highest fields reached.

\footnotetext{
${ }^{1}$ Note that, in contrast to the described situation, in semiconductors with a very low ionization energy of impurities, magnetic field resulting in freezing-out of free carriers creates preconditions for appearance of impact ionization [9].
}

\section{(C) 2012, V. Lashkaryov Institute of Semiconductor Physics, National Academy of Sciences of Ukraine}




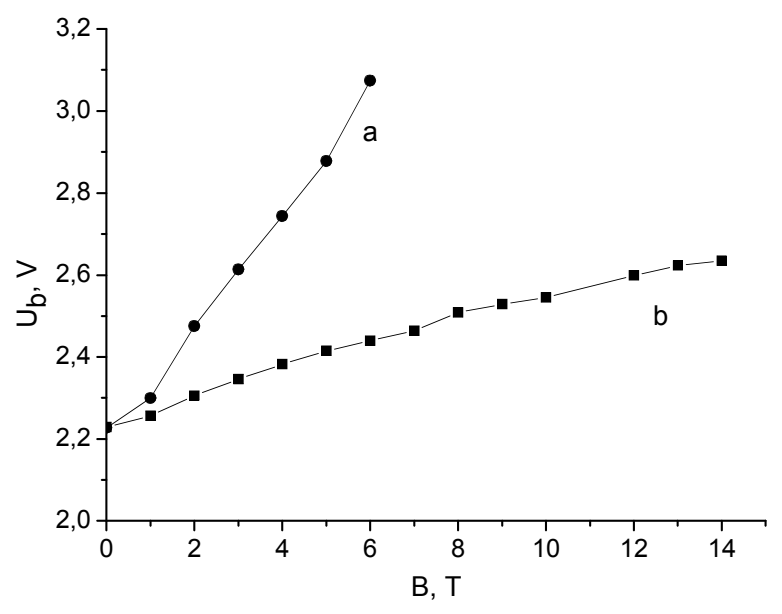

Fig. 2. Dependences of the threshold breakdown voltages on the magnetic field; (a), (b) are the same as in Fig. 1.

The values of breakdown voltages for both magnetic field orientations are shown in Fig. 2 as a function of magnetic induction $B$. A qualitatively similar picture was observed in Ge samples (with negligible hysteresis loops) [10].

\section{Analysis of the experimental results}

\subsection{Determination of breakdown electric fields in the diode base}

The breakdown voltages represented in Fig. 2 are the total diode voltages. For quantitative analysis of ionization processes in the freezed-out base of the diode, it is necessary to separate out (in the total voltage) a portion of the voltage drop across the base. Thereto, we used our previously developed method of extraction from diode CVC of the series resistance generated under freezing-out of the diode base $[11,12]$. As it was shown in [12] (where the same diode has been analyzed) the voltage drop across the base under the breakdown at $4.2 \mathrm{~K}$ constituted $1.18 \mathrm{~V}$; meanwhile, the total voltage drop across the diode was $2.4 \mathrm{~V}$. The difference of $1.22 \mathrm{~V}$ which falls on the $n^{+}-p$ junction was subtracted from all the points in Fig. 2.

The breakdown voltages $U_{\text {base }}$ of the base obtained in this way (as a function of magnetic induction) were used then for calculation of the electric field in the base under breakdown. Due to circular symmetry of the diode (Fig. 3), the electric field in the base is a function of radius and its voltage drop $E_{1}=\frac{U_{\text {base }}}{\ln \left(r_{2} / r_{1}\right)} \frac{1}{r_{1}}$, when the inner radius $r_{1}$ is maximal. It was this value that was used for analysis of impact ionization in the diode base. Calculated dependences of the breakdown electric fields $E_{b}$ on magnetic induction $B$ are shown for both magnetic field orientations in Fig. 4 (as experimental points).

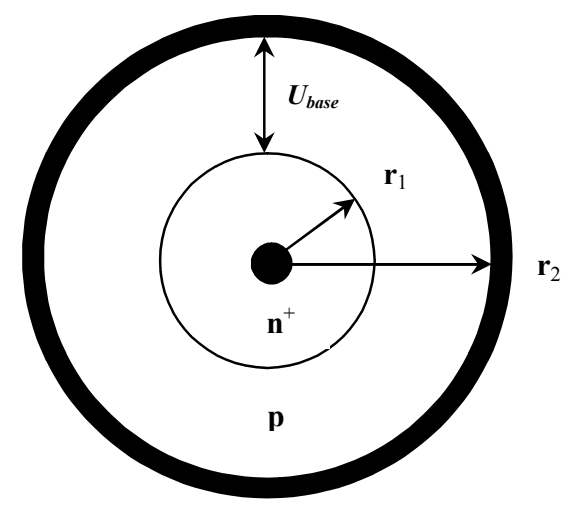

Fig. 3. Scheme of the diode structure in plan.

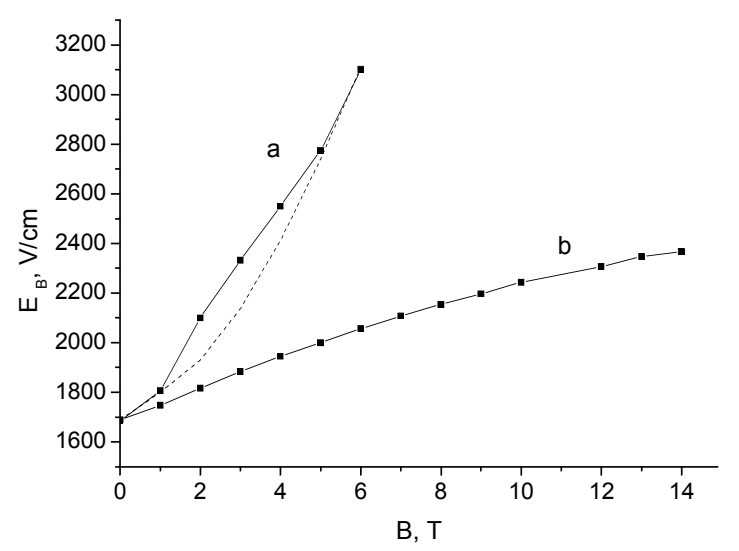

Fig. 4. Dependences of the breakdown electric fields in the diode base on the magnetic field; (a), (b) are the same as in Fig. 1.

\subsection{Determination of impurity ionization energy depending on magnetic field}

Semi-empirical theory [13] for impact ionization of shallow impurities in silicon gives the following expression for the impact ionization coefficient:

$A_{i i}(E)=$ const $\frac{1}{x^{3 / 2}} \exp \left(-\frac{1.33}{x}\right)$,

where $E$ is the electric field, $x=\left[(\alpha+1 / 2) E^{2} m \mu^{2}+k T_{0}\right] / \varepsilon_{i}, \quad m-$ effective mass of carriers executing impact ionization, $\mu(E)$ - their mobility, $k$ - the Boltzmann constant, $T_{0}$ - lattice temperature, $\varepsilon_{i}$ - ionization energy of the impurity center depending on the magnetic field. The parameter $\alpha$ describes increase in the effective carrier temperature $T_{\text {eff }}$ at the expense of its drift motion in electric field: $k T_{e f f}=k T_{0}+\alpha m v_{d}^{2}$. 
At small values of $x$, the coefficient $A_{i i}(E)$ is exponentially small. Therefore, it is naturally to believe that impact ionization takes place at $x$ close to 1 . In presence of the magnetic field $B$, the condition $x=1$ takes the form

$\left(\alpha+\frac{1}{2}\right) E_{b}^{2}(B) m \frac{\mu^{2}}{\left[1+(\mu B)^{2}\right]^{2}}+k T_{0}=\varepsilon_{i}(B)$,

where $E_{b}(B)$ is the breakdown electric field and influence of magnetic field on both electron mobility $\mu$ and ionization energy $\varepsilon_{i}$ has been taken into account. The phenomenological dependence of electron mobility on the electric field was used in the form $\mu=\mu_{0} /\left(1+\mu_{0} E / v_{\text {sat }}\right)$, where $\mu_{0}$ is the mobility in low electric fields, $v_{\text {sat }}$ is the saturation drift velocity equal to $10^{7} \mathrm{~cm} / \mathrm{s}$ for $\mathrm{Si}$ [13].

Under perpendicular orientation of the magnetic field, the effect of curling electron trajectories is the main one. The obtained from (2) dependence of the breakdown electric field $E_{b}$ on the magnetic field induction $B$ (at $\varepsilon_{i}=$ const $=0.025 \mathrm{eV}^{2}$ and $m=0.26 m_{0}$ ) agrees in the best way with experimental points at $\mu_{0}=$ $1840 \mathrm{~cm}^{2} / \mathrm{V} \cdot \mathrm{s}$ and $\alpha+\frac{1}{2}=28.4$ (dashed curve in Fig. 4).

Under parallel orientation of magnetic field, the electron mobility does not vary with magnetic field. For this case, the equation (2) takes the form

$\left(\alpha+\frac{1}{2}\right) E_{b}^{2}(B) m\left(\frac{\mu_{0}}{1+\mu_{0} E_{b} / v_{s a t}}\right)^{2}+k T_{0}=\varepsilon_{i}(B)$,

wherefrom, using the experimental dependence $E_{b}(B)$ for parallel orientation and abovementioned values of $\mu_{0}$ and $\alpha+\frac{1}{2}$, the dependence of $\varepsilon_{i}(B)$ can be determined. It is shown in Fig. 5.

It follows from Fig. 5 that the ionization energy of boron impurity in silicon (at a given doping level) increases in magnetic field of $14 \mathrm{~T}$ by the factor close to 1.54. Existing theoretical calculations of this dependence has been carried out in the framework of the hydrogenic impurity model with the scalar effective mass and parabolic dispersion law of carriers in the bands. In particular, results of these calculations have been represented [14] in the form of the dependence of an impurity center ground state on the dimensionless parameter $\gamma=\frac{\hbar \omega_{c}}{2 R}$ that is the ratio of the characteristic carrier energy in the lowest Landau band $\hbar \omega_{c} / 2$ to the

${ }^{2}$ Value of the ionization energy used here is equal to the activation energy of Hall effect in silicon resistor identical completely (in its properties) to the base layer of the investigated diode. energy of effective Rydberg $R=m_{h} e^{4} / 2 \hbar^{2} \kappa^{2}$ for the acceptor center. Here, $\omega_{c}$ is the cyclotron frequency, $\hbar$ - Plank constant (divided by $2 \pi$ ), e - elementary charge, $\kappa-$ dielectric constant. In the given case, at $B=$ $14 \mathrm{~T} \gamma=0.34$ (the light hole effective mass of $0.16 m_{0}$ is used here). According to the theory [14], increase in the ground state energy by the factor 1.29 corresponds to this $\gamma$ value that is somewhat less than it is follows from the analysis of the experimental data. The reasons of disagreement are, obviously, both roughness of the analysis and imperfection of the theory. Though the results of analysis demonstrate the commonly observed in similar cases [15-17] dependence on the magnetic field of $B^{1 / 3}$-type (the insert in Fig. 5).

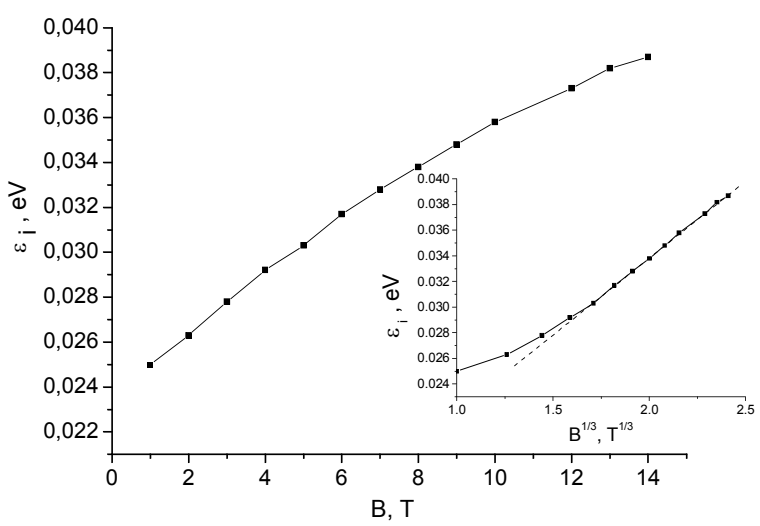

Fig. 5. Calculated from the experimental data dependence of the ionization energy of boron impurity in silicon (at given doping level) on the magnetic field.

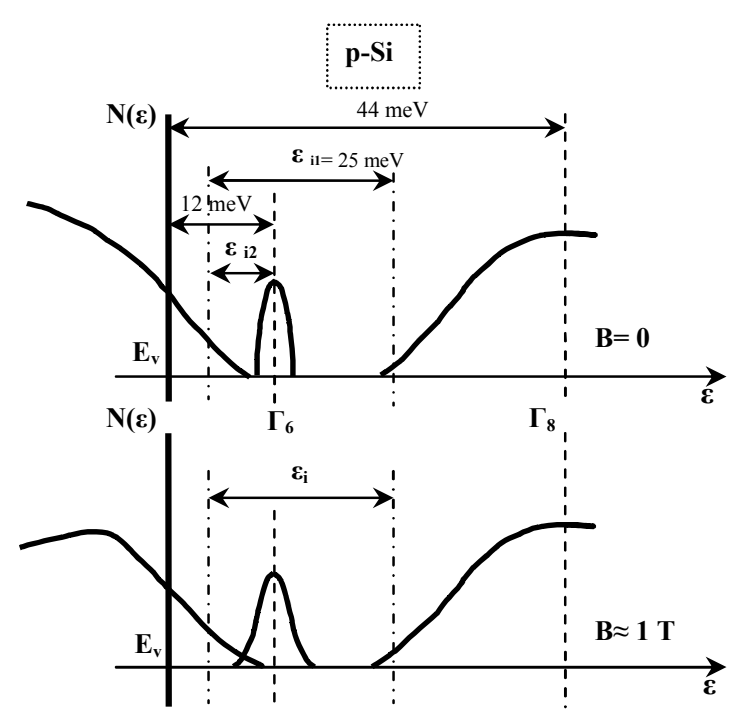

Fig. 6. Model for the density of states in the diode base which explains a qualitatively possible mechanism providing suppression of the hysteresis loops by magnetic field. 


\subsection{Interpretation of suppression of the hysteresis loops by magnetic field}

Generally accepted treatment of the hysteresis mechanism in low-temperature impurity breakdown is based on taking into account (in ionization and recombination processes) another (one) middle energy level - as a rule, of the first excited state inherent to the impurity center. Impact ionization of the carriers accumulated on this level takes place at a lower applied voltage. This fact results in existence of holding electric field.

However, in our case, due to a high enough doping level (close to IMT) all the energy levels are substantially fuzzy. Theoretical analysis of the fluctuation potential amplitude was carried out within the limits of light doping and heavy doping [18]. Concentration region near the IMT but on its insulator side, as far as we know, has not been considered theoretically. We used here, for assessment of the fluctuation potential amplitude $\Gamma$, more suitable for our case, in our view, formulas of the heavy-doping limit when $\Gamma=2 \sqrt{\pi} \frac{e^{2}}{\kappa r_{0}}\left(N_{A} r_{0}^{3}\right)^{1 / 2}$ and $r_{0}^{2}=\frac{\kappa k T}{4 \pi e^{2} N_{A}}$. At $N_{A}=3 \cdot 10^{18} \mathrm{~cm}^{-3}$ (doping level of the diode base), we obtain $\Gamma \approx 12 \mathrm{meV}$ that is of the same order of magnitude as the ionization energy of the first excited state of boron in silicon, which is assumed to be spreadout as well. Therefore, there is a rather narrow gap in this place of the density of states (Fig. 6, top picture) which can be responsible for existence of the second (lower) ionization energy and, as consequence, of the holding voltage.

Under application of magnetic field, both states of the first excited level and localized states in the tail of the density of states in the valence band suffer Zeeman effect, which, in principle, can close the abovementioned gap (Fig. 6, bottom picture). Thereby, the lower ionization energy is eliminated, and it also eliminates hysteresis.

As one of arguments for this model, one can point to results of the paper [19], where authors have measured Zeeman effect in $\mathrm{Si}(: \mathrm{B})$ for different orientations of magnetic field relatively to crystallographic axes. When $\mathrm{B} \|[111]$, the effect was substantially less than for other field orientations. In our case, the effect of magnetic field on the hysteresis loops is less just under perpendicular orientation of the magnetic field relatively to the (111)-oriented diode structure, i.e. for $\mathrm{B} \|[111]$ direction.

\section{Conclusion}

So, although silicon is the main material of semiconductor electronics, dependence of the ionization energy of boron impurity in it on magnetic field, as far as we know, was not determined so far, all the more - at high doping level (close to Mott transition). And the suppression of the hysteresis loops in the diode CVC by magnetic field can be qualitatively explained by Zeeman effect on localized states taking into account a fluctuation potential connected with heavy doping.

\section{References}

1. R.V. Aldridge, K. Davis, and M. Holloway, An investigation of the effect of a magnetic field on the forward characteristics of some silicon diodes at low temperatures // J. Phys. D, 8(1), p. 64-68 (1975).

2. E. Simoen, B. Dierickx, L. Deferm, and C. Claeys, The behavior of silicon $p$ - $n$ junction based devices at liquid helium temperatures // J. Appl. Phys. 70(2), p. 1016-1024 (1991).

3. B. Dierickx, L. Warmerdam, E. Simoen, J. Wermeiren, and C. Claeys, Model for hysteresis and kink behavior of MOS transistors operating at $4.2 \mathrm{~K} / /$ IEEE Trans. ED-35(7), p. 1120-1125 (1988).

4. R. Richter, A. Kittel, G. Heinz, G. Flatgen, J. Peinke, and J. Parisi, Type-I intermittency in semiconductor breakdown: An experimental confirmation // Phys. Rev. B, 49(13), p. 8738-8746 (1994).

5. J. Spangler, U. Margull, and W. Prettl, Regular and chaotic current oscillations in n-type GaAs in transverse and longitudinal magnetic fields // Phys. Rev. B, 45(20), p. 12137-12140 (1992).

6. S-Y.T. Tzeng and Y. Tzeng, Two-level model of longitudinal magnetic field-induced current instability and chaos in n-GaAs // Phys. Rev. B, 72, 205201(1-7), (2005).

7. K. Aoki, T. Kondo, and T. Watanabe, Cross-over instability and chaos of hysteretic I-V curve during impurity avalanche breakdown in $\mathrm{n}-\mathrm{GaAs}$ under longitudinal magnetic field // Solid State Communs. 77(1), p. 91-94 (1991).

8. V.A. Samuilov, V.K. Ksenevich, G. Remenyi, G. Kiss, and B. Podor, Impact ionization breakdown of $\mathrm{n}-\mathrm{GaAs}$ in high magnetic field // Semicond. Sci. Technol. 14(12), p. 1084-1087 (1999).

9. R.J. Phelan and W.F. Love, Negative resistance and impact ionization impurities in n-type indium antimonide // Phys. Rev. 133(4A), p. A1134-A1137 (1964).

10. T.O. Poehler and J.R. Apel, Impurity ionization in germanium in strong magnetic fields // Phys. Rev. $B$, 1(8), p. 3240-3244 (1970).

11. V.L. Borblik, Yu.M. Shwarts, M.M. Shwarts, A new method of extraction of a $p-n$ diode series resistance from $I-V$ characteristics and its application to analysis of low-temperature conduction of the diode base // Semiconductor Physics, Quantum Electronics \& Optoelectronics, 12(3), p. 339-342 (2009). 
12. V.L. Borblik, Yu.M. Shwarts, M.M. Shwarts, and A.M. Fonkich, Concerning the nature of relaxation oscillations in silicon diodes in the cryogenic temperature region // Cryogenics. 50(6-7), p. 417420 (2010).

13. B. Dierickx, E. Simoen, and G. Declerck, Transient response of silicon devices at $4.2 \mathrm{~K}$ : I. Theory // Semicond. Sci. Technol. 6(9), p. 896-904 (1991).

14. D.M. Larsen, Shallow donor levels of InSb in a magnetic field // J. Phys. Chem. Sol. 29(2), p. 271280 (1968).

15. O. Beckman, E. Hanamura, and L.J. Neuringer, Quantum limit galvanomagnetic phenomena in nInSb // Phys. Rev. Lett. 18(19), p. 773-775 (1967).
16. L.A. Kaufman and L.J. Neuringer, Magnetic freeze-out and band tailing in n-InAs // Phys. Rev. $B, 2(6)$, p. 1840-1846 (1970).

17. T.O. Poehler, Magnetic freeze-out and impact ionization in GaAs // Phys. Rev. B, 4(4), p. 12231229 (1971).

18. B.I. Shklovskii and A.L. Efros, The Electronic Properties of Doped Semiconductors. Nauka, Moscow, 1979 (in Russian).

19. F. Merlet, B. Pajot, Ph. Arcas, and A.M. JeanLouis, Experimental study of the Zeeman splitting of boron levels in silicon // Phys. Rev. B, 12(8), p. 3297-3317 (1975). 